\title{
Fenótipo comportamental de crianças e adolescentes com síndrome de Prader-Willi
}

\author{
Behavioral phenotype of children and adolescents with Prader-Willi syndrome
}

\author{
Maria Luiza G. de Mesquita1 , Décio Brunoni², João Monteiro de Pina Neto ${ }^{3}$, Chong Ae Kim ${ }^{4}$, Márcia Helena S. Melo 5 , \\ Maria Cristina T. V. Teixeira ${ }^{6}$
}

\section{RESUMO}

Objetivo: Identificar, em um grupo de crianças e adolescentes com síndrome de Prader-Willi, as principais características do fenótipo comportamental.

Métodos: A amostra foi composta por 11 crianças e adolescentes com diagnóstico clínico e citogenéticomolecular da síndrome de Prader-Willi. A técnica de coleta de dados foi o Inventário dos Comportamentos de Crianças e Adolescentes entre 6 e 18 anos (CBCL/6-18). $A$ análise de correlação bivariada, com nível de significância $p<0,05$, foi usada para testar a associação entre as variáveis analisadas.

Resultados: Os principais resultados mostraram um perfil comportamental considerado como clínico em várias das escalas do CBCL/6-18. Observou-se um padrão comportamental com alta frequência de respostas de agressão, quebra de regras e oposição. Identificaram-se correlações estatisticamente significativas entre problemas de atenção e sociais e problemas de pensamento e comportamento de quebrar as regras.

Conclusões: Os pacientes investigados representam um grupo com risco psiquiátrico e alterações de comportamento que, em longo prazo, poderão evoluir para transtornos de humor, do déficit de atenção e hiperatividade e transtorno desafiador e de oposição, dentre outros.

Palavras-chave: síndrome de Prader-Willi; comportamento; saúde mental.

\section{ABSTRACT}

Objective: To identify the main characteristics of the behavioral phenotype of children and adolescents with Prader-Willi syndrome.

Methods: Eleven children and adolescents with clinical and cytogenetic-molecular diagnosis of Prader-Willi syndrome were studied. Data collection was obtained by the Child Behavior Checklist for Ages 6-18 (CBCL/6-18). Bivariate correlations were used to test the association between the analyzed variables, being significant $\mathrm{p}<0.05$.

Results: The behavioral profile obtained was considered as clinical in different scales of the CBCL/6-18 tool. A behavioral pattern with high frequency of aggression, rule breaking and opposition was observed. Statistically significant correlations between attention and social problems and between thought problems and breaking rules were identified.
Instituição: Programa de Pós-Graduação em Distúrbios do Desenvolvimento do Centro de Ciências Biológicas e da Saúde da Universidade Presbiteriana Mackenzie (Mackenzie), São Paulo, SP, Brasil

'Mestre em Distúrbios de Desenvolvimento pelo Programa de PósGraduação em Distúrbios do Desenvolvimento do Mackenzie, São Paulo, SP, Brasil

2Doutor em Ciências Biológicas pela Universidade Federal do Rio de Janeiro; professor titular do Programa de Pós-Graduação em Distúrbios do Desenvolvimento do Mackenzie, São Paulo, SP, Brasil

${ }^{3}$ Doutor em Genética pela Universidade de São Paulo (USP); professor titular da Faculdade de Medicina de Ribeirão Preto da USP, Ribeirão Preto, SP, Brasil

${ }^{4}$ Doutor em Medicina pela USP; professor livre-docente pela USP; chefe da Unidade de Genética do Instituto da Criança do Departamento de Pediatria do Hospital das Clínicas da Faculdade de Medicina da USP, São Paulo, SP, Brasil

${ }^{5}$ Doutora e Pós-Doutora em Psicologia Clínica pela USP; colaboradora do Laboratório de Terapia Comportamental da USP, São Paulo, SP, Brasil
${ }^{6}$ Doutora em Saúde pela Universidade Federal de Santa Catarina; professor adjunto do Programa de Pós-Graduação em Distúrbios do Desenvolvimento do Programa de Pós-Graduação em Distúrbios do Desenvolvimento do Mackenzie, São Paulo, SP, Brasil

Endereço para correspondência:

Maria Cristina T. V. Teixeira

Rua da Consolação, 896, Prédio 38, Térreo - Consolação

CEP 01302-907 - São Paulo/SP

E-mail: cris@teixeira.org

Fonte financiadora: Instituto Presbiteriano Mackenzie/MackPesquisa Conflito de interesse: nada a declarar

Recebido em: 8/4/2009

Aprovado em: 23/7/2009 
Conclusions: The studied patients represent a group of psychiatric risk, with behavioral changes that, in the long-term, can lead to mood disorders, attention deficit hyperactivity disorder and oppositional defiant behavior, among other disorders.

Key-words: Prader-Willi syndrome; behavior; mental health.

\section{Introdução}

A síndrome de Prader-Willi (SPW) é uma doença genética cuja causa decorre da falta de expressão dos genes da região cromossômica 15q11-q13 paterna, que sofrem o fenômeno de imprinting genômico. Genes desta região têm expressão diferencial de acordo com a origem parental, de modo que as cópias paterna e materna devem estar presentes para a expressão gênica normal. Os critérios para diagnóstico da SPW estão bem definidos e o diagnóstico laboratorial é estabelecido em $95 \%$ dos casos: em $70 \%$ dos pacientes há a presença da deleção de 15q11-q13 paterna e $25 \%$ têm dissomia uniparental (os dois cromossomos 15 são de origem materna e nenhum de origem paterna). A incidência da doença é 1:10.000 a 15.000 nascimentos ${ }^{(1)}$.

A SPW caracteriza-se por duas fases clínicas. Na primeira, os sintomas cardinais são: hipotonia neonatal, dificuldade de alimentação, letargia, choro fraco e hiporreflexia. A segunda, a partir dos seis meses, apresenta melhora gradual da hipotonia, ganho de peso e desenvolvimento progressivo da hiperfagia e obesidade. Alterações genitais como criptorquidismo, micropênis e bolsa escrotal hipoplásica são encontradas em crianças do sexo masculino e, hipoplasia dos genitais externos, no sexo feminino ${ }^{(2)}$. Crianças com obesidade de outras causas não apresentam complicações respiratórias significativas, já indivíduos com SPW podem ter problemas ventilatórios fatais ${ }^{(3)}$.

Diversas alterações de comportamento na infância, adolescência e vida adulta ocorrem: comportamentos do espectro obsessivo-compulsivo, crises de raiva e violência, tendência à rigidez, condutas de oposicionismo, comportamentos de mentir e furtar, deficiência mental leve a moderada, alterações neuropsicológicas associadas com a atividade do lobo frontal no que concerne à atenção, habilidades executivas e organização visoespacial ${ }^{(4-13)}$. Muitas das manifestações patológicas de natureza comportamental se associam à falta de controle inibitório de base biológica frente a diversos estímulos, dentre eles os alimentares e a restrição dos pais para evitar ingestão calórica exagerada ${ }^{(14-16)}$.

As queixas psicológicas mais frequentes no quadro de hiperfagia são alterações de comportamento e transtornos psiquiátricos $^{(11,17,18)}$. O estudo de alterações de comportamento na SPW tem sido objeto de pesquisa há vários anos. Doenças psiquiátricas, como transtorno do humor, transtornos psicóticos, alterações em habilidades de competência social, problemas somáticos e de hiperatividade, com déficit de atenção, foram relatados em crianças com SPW ${ }^{(17,18)}$. Existem trabalhos que compararam as alterações comportamentais destas com o de crianças de outros grupos, sendo os escores médios de problemas de comportamento maiores no grupo com SPW com relação a, por exemplo, problemas sociais, de pensamento, atenção, comportamento delinquente, agressivo e de quebrar regras ${ }^{(16)}$.

No Brasil, observa-se uma escassez de trabalhos focando as áreas de avaliação de saúde mental e intervenção comportamental na SPW, assim como estudos direcionados ao desenvolvimento de programas de prevenção de algumas destas alterações. Assim, a presente pesquisa teve como objetivo identificar, em um grupo crianças e adolescentes com SPW, as principais características do fenótipo comportamental.

\section{Métodos}

Descrição de uma série de 11 crianças e adolescentes com diagnóstico clínico e citogenético-molecular da SPW. Os critérios de inclusão foram: confirmação em prontuário de diagnóstico clínico e citogenético-molecular da SPW e idade entre seis e 18 anos. Todos os pacientes foram selecionados do Serviço de Genética da Faculdade de Medicina da Universidade de São Paulo, Ribeirão Preto e do Serviço de Genética do Instituto da Criança da Faculdade de Medicina da Universidade de São Paulo. A pesquisa teve aprovação do Comitê de Ética da Universidade Presbiteriana Mackenzie.

Foram coletadas, em um período de quatro meses, as seguintes informações dos prontuários: mecanismo do erro genético; sexo; dados a respeito de outros diagnósticos psiquiátricos, uso de medicação psicotrópica e acompanhamento psiquiátrico. Quando estes últimos não se encontravam atualizados nos prontuários, os dados foram coletados junto às mães. A idade mediana do grupo era de 12 anos e, quanto à escola, seis frequentavam instituições de ensino regular $\mathrm{e}$ cinco, escolas especiais. A média de índice de massa corporal do grupo foi 31,96, valor compatível com obesidade, segundo Must, Dallal e Dietz ${ }^{(19)}$. 
Quanto à coleta de dados para o objetivo do presente estudo, primeiro realizou-se entrevista com a mãe para identificar padrões de interação familiar associados ao comportamento alimentar e reações ao controle alimentar imposto pelos pais, para coleta de dados sobre diagnóstico psiquiátrico, acompanhamento psiquiátrico, uso de medicações psicotrópicas e tipo de escola frequentada. A seguir, foi feita a avaliação comportamental por meio do Inventário dos Comportamentos de Crianças e Adolescentes entre 6 e 18 anos (CBCL/6-18) de Achenbach e Rescorla ${ }^{(20)}$. A avaliação foi efetuada a partir das respostas das mães sobre os comportamentos das crianças e adolescentes (competência social e problemas de comportamento) nos seis meses que antecederam a aplicação do instrumento. Ressalta-se que o CBCL/6-18 é um questionário de avaliação comportamental de utilização internacional, desenvolvido para obter dados de percepção dos pais sobre competência social e problemas de comportamento de seus filhos.

Os dados do CBCL/6-18 foram registrados no programa de computador Assessment Data Manager 7.2 para gerar perfis comportamentais. Os escores obtidos foram distribuídos nos perfis: clínico, limítrofe e não-clínico. Essa classificação indica a necessidade ou não de as crianças e adolescentes receberem acompanhamento psicológico e/ou pedagógico ${ }^{(20)}$. Para avaliar correlações entre os diferentes escores da escala, conduziram-se análises descritivas de médias nas escalas e a análise de correlação bivariada, por meio do coeficiente de correlação Spearman, com nível de significância de 5\%.

\section{Resultados}

$\mathrm{Na}$ Tabela 1 são sumarizados os dados gerais dos sujeitos em relação à idade, ao sexo, ao tipo de escola que frequentam/escolaridade, diagnóstico molecular, diagnóstico e/ou acompanhamento psiquiátrico, uso de medicação psicotrópica e índice de massa corporal. Vale destacar que, em relação ao diagnóstico molecular, observou-se um predomínio do subtipo genético deleção cromossômica (em nove de 11 pacientes). Os outros dois (18\%) apresentavam o mecanismo da dissomia uniparental materna. Nove sujeitos faziam uso de diversos tipos de psicotrópicos, sendo a risperidona o neuroléptico mais utilizado (três crianças). Em ordem decrescente de uso, os seguintes fármacos antidepressivos foram citados: clomipramina, fluoxetina e os estabilizadores de humor topiramato e fenobarbital.
Não constavam nos prontuários dados sobre diagnóstico psiquiátrico e uso de medicação. No entanto, conforme mostra a Tabela 1, os responsáveis informaram o uso de fármacos sem qualquer acompanhamento psiquiátrico, incluindo o único caso em que o transtorno obsessivo-compulsivo parecia estar confirmado de acordo com o dado do informante. Quanto à escolaridade, a maioria das crianças frequentava o ensino especial, enquanto quatro frequentavam o ensino regular nas séries terceira, quarta, quinta e sétima. Ao comparar a idade com a série dessas quatro crianças, observa-se incompatibilidade, evidenciando a repetência escolar e dificuldades para acompanhar o processo de alfabetização.

Ao avaliar as escalas de competências em sociabilidade, área escolar e realização de atividades obtidas no CBCL, a escala 'social,' que avalia essencialmente o número de colegas e as organizações sociais que a criança frequenta, obteve pontuação na faixa da normalidade (média $=38,5$ pontos). Por outro lado, as habilidades das crianças para a realização de 'atividades' que incluam esportes e lazer (média $=30,8$ pontos) e 'desempenho escolar' (média $=28,3$ pontos) pontuaram, respectivamente, na faixa limítrofe e clínica.

Os escores T médios das escalas das síndromes são mostrados na Figura 1. Na faixa limítrofe, encontram-se os problemas comportamentais ('isolamento/depressão') e 'problemas sociais', explicados, dentre outros, pela excessiva dependência de adultos, solidão, ciúmes e dificuldades no relacionamento social. As escalas que pontuaram na faixa clínica foram 'comportamento agressivo', 'problemas sociais' e 'problemas de pensamento’, cujos padrões de alteração comportamental são ideias obsessivas, comportamentos autolesivos, alucinações, tiques, alterações do sono, desobediência, heteroagressividade e impulsividade.

Quatro das seis escalas do CBCL/6-18, orientadas pelo Manual Diagnóstico e Estatístico de Transtornos Mentais ${ }^{(21)}$ pontuaram na faixa limítrofe (problemas afetivos com 67,5 pontos; problemas de déficit de atenção com hiperatividade com 65,1 pontos; comportamento de desafio e oposição com 65 pontos e problemas de conduta resultando em 67,7 pontos). As duas escalas restantes foram classificadas na faixa normal (problemas somáticos, 59,5 pontos e problemas de ansiedade, 60,3 pontos).

Com a finalidade de avaliar a existência de correlação entre os escores $\mathrm{T}$ das síndromes e os escores do funcionamento adaptativo, executou-se a análise de correlação bivariada com o coeficiente de correlação Spearman. Identificaram-se coeficientes de correlação negativos e estatisticamente significativos entre: problemas de pensa- 
Tabela 1 - Características clínicas dos pacientes estudados

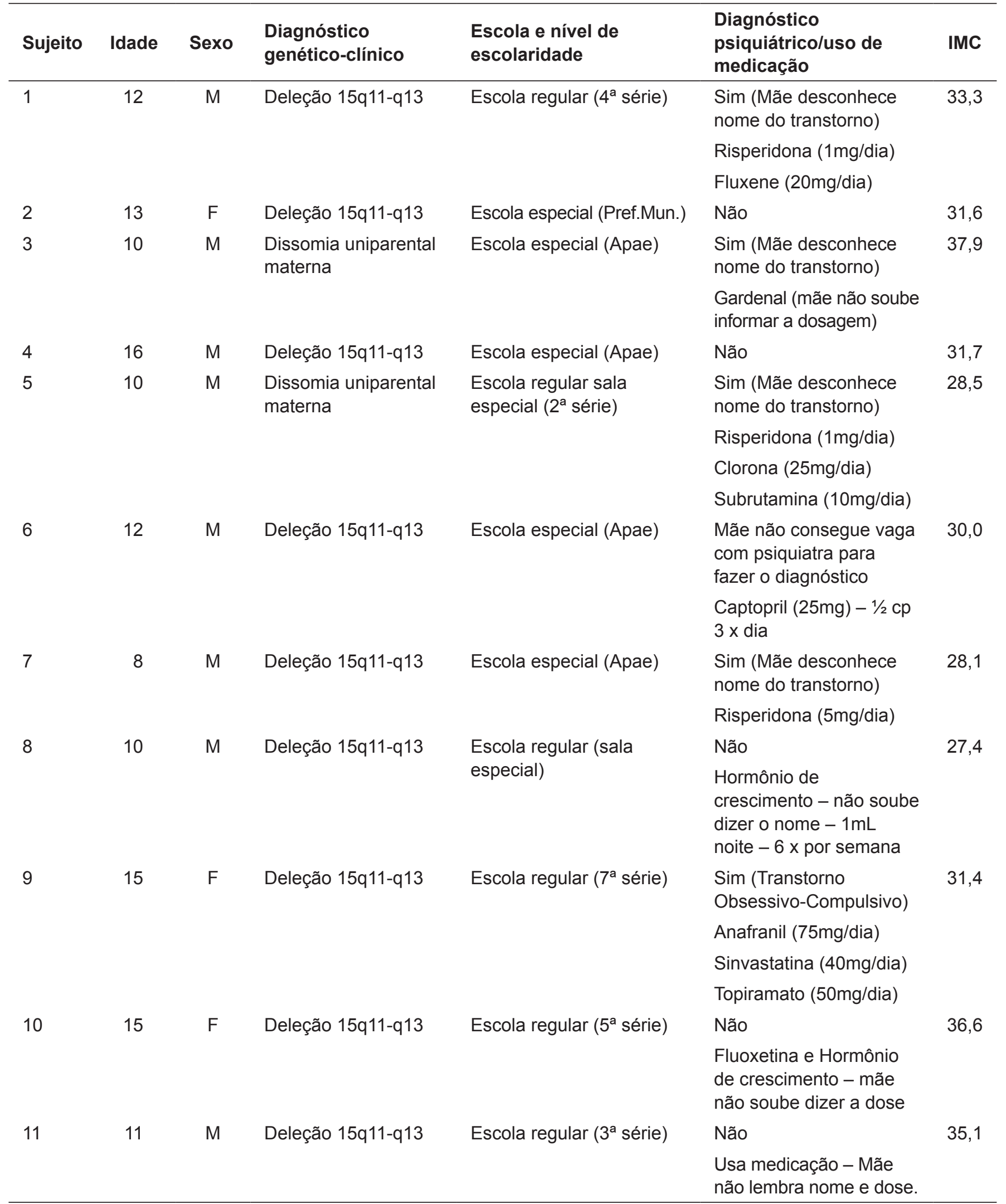

IMC: índice de massa corporal; M: masculino; F: feminino 
mento e desempenho de atividades ( $\mathrm{Rho}=0,69, p=0,01$ ); comportamento de quebrar regras e desempenho de atividades ( $\mathrm{Rho}=0,71, p=0,02)$; e competências de desempenho escolar e problemas sociais ( $\mathrm{Rho}=0,81, p=0,01$ ). Os coeficientes de correlação positivos e estatisticamente significativos se apresentam entre as escalas: isolamento, depressão e problemas sociais ( $\mathrm{Rho}=0,61, p=0,04)$; problemas de atenção e sociais ( $\mathrm{Rho}=0,72, p=0,01$ ); problemas de pensamento e atenção ( $\mathrm{Rho}=0,69, p=0,01)$; problemas de pensamento e comportamento de quebrar regras ( $\mathrm{Rho}=0,87, p=0,001)$; problemas de pensamento e agressividade ( $\mathrm{Rho}=0,68, p=0,01)$.

Dados da entrevista sobre os padrões de interação familiar associados ao comportamento alimentar apontaram que $100 \%$ dos sujeitos furtavam alimentos e comiam escondidos; $73 \%$ das mães relataram que os participantes sempre tinham acesso aos locais onde se guardavam os alimentos. As mães referiram que, nas primeiras tentativas de obter o alimento, as crianças e adolescentes se mostravam carinhosos e afetuosos, entretanto essa afetuosidade mudava nas tentativas seguintes. Assim, a frequência média de comportamentos das crianças na espera do alimento (que sempre era obtido) acontecia na seguinte ordem: comportamentos socialmente habilidosos e assertivos ( $n=11)$, negociação $(n=9)$, suborno $(n=9)$, birra $(n=9)$, agredir $(n=9)$, furtar $(n=11)$ e pedir alimentos na vizinhança $(\mathrm{n}=11)$.

\section{Discussão}

O instrumento CBCL/6-18 tem sido usado de maneira confiável em diversos estudos para descrever perfis comportamentais de crianças com diversos transtornos do desenvolvimento e mentais ${ }^{(11,22,23)}$. Trabalhos futuros permitirão estudos mais acurados deste perfil da amostra, com a utilização de outros questionários do Sistema de Avaliação Empiricamente Baseado (ASEBA), por exemplo, os de autorrelato e os que são aplicados a outros informantes.

Os resultados do estudo nas escalas de competências coincidem com pesquisas anteriores que também destacam as dificuldades de pacientes com SPW em realizar atividades, caminhadas e exercícios físicos ${ }^{(24)}$. O baixo aproveitamento escolar identificado associa-se com possíveis alterações neuropsicológicas já apontadas por outros autores, a saber, déficit de atenção e prejuízos na memória operacional e habilidades executivas ${ }^{(9,18)}$. Houve correlações estatisticamente significativas entre problemas de funcionamento adaptativo e outras escalas

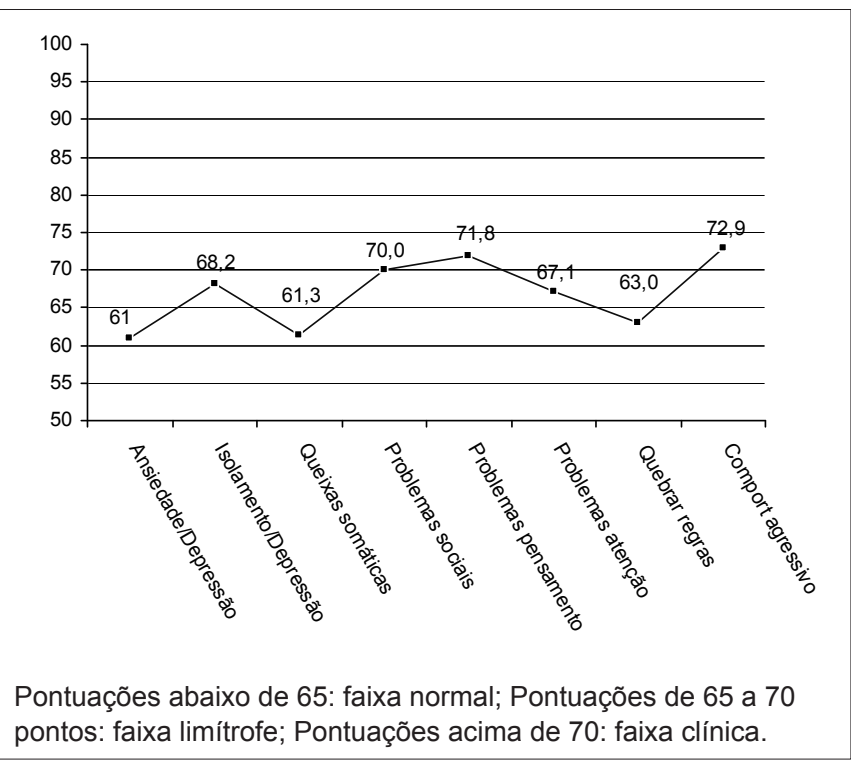

Figura 1 - Médias de escores T do CBCL/6-18.

avaliadas pelo CBCL/6-18, por exemplo, desempenho de atividades, problemas sociais, comportamento agressivo e comportamento de quebrar regras. Isso permite inferir que, de um lado, são crianças que mantêm relações sociais razoáveis em termos de frequência (mostrado na classificação de normalidade na escala social de competências). De outro, há alterações de comportamento refletidas, por exemplo, na qualidade desses relacionamentos a partir de relações de dependência com adultos, solidão, ciúmes e problemas nas relações sociais, dentre outros (pontuações clínicas obtidas na escala problemas sociais).

Os escores não-clínicos obtidos nas escalas das síndromes (ansiedade/depressão, queixas somáticas e comportamento de quebrar regras) diferem dos achados de outros autores ${ }^{(25,26)}$. Por exemplo, estudos anteriores mostram que comportamentos de quebrar regras em pacientes com SPW se associam frequentemente à procura de alimentos ${ }^{(1,26)}$. No presente estudo, $73 \%$ das mães relataram que os pacientes sempre têm acesso aos locais onde os alimentos são estocados. É provável que este seja um dos fatores que contribuiu para obter escores não-clínicos na escala relativa aos comportamentos de quebrar regras. Aliás, assim como ocorre em crianças com desenvolvimento normal e obesidade ${ }^{(27)}$, o livre acesso aos lugares onde se guardam alimentos também pode contribuir para o aumento de obesidade nas crianças e adolescentes pesquisados.

Manifestações comportamentais encontradas neste grupo fazem parte das alterações de comportamento que caracteri- 
zam o chamado fenótipo comportamental da SPW (problemas sociais, comportamentos autolesivos e comportamentos agressivos). O termo fenótipo comportamental refere-se àquele padrão cognitivo e comportamental típico de uma síndrome cuja causa se deve a doenças de origem genética e a fatores ambientais ${ }^{(11,18)}$. O cunho biologicista do termo fenótipo comportamental aplicado a doenças genéticas pertence ao pesquisador Nyhan que, em 1972, descreveu comportamentos autolesivos de crianças com déficit de hipoxantinaguanina fosforibosil transferase. Atualmente, os pesquisadores reconhecem a influência de fatores biológicos associados a anormalidades genéticas, porém não subestimam os efeitos do ambiente nas alterações de comportamento ${ }^{(28)}$.

Os achados clínicos em diversos comportamentos das escalas das síndromes e limítrofes em três escalas, orientadas pelo Manual Diagnóstico e Estatístico de Transtornos Mentais, conforme normas do manual do $\mathrm{CBCL} / 6-18^{(20)}$, indicam a necessidade de acompanhamento psicológico el ou pedagógico desta série de casos. Apesar de o número de indivíduos estudados ser restrito, a elevada frequência de alterações de comportamento indica que tais crianças e adolescentes poderão evoluir, em longo prazo, para transtornos de humor, transtorno do déficit de atenção e hiperatividade e transtorno desafiador e de oposição, dentre outros, como tem sido comprovado em estudos com adultos ${ }^{(1,8,13)}$.

Pode-se concluir que, embora o número amostral seja não-probabilístico, este estudo é o primeiro trabalho brasileiro que descreve o fenótipo comportamental de crianças e

\section{Referências bibliográficas}

1. Cassidy SB, Driscoll DJ. Prader-Willi syndrome. Eur J Hum Genet 2009;17: 3-13.

2. Holm VA, Cassidy SB, Butler MG, Hanchett JM, Greenswag LR, Whitman BY et al. 1993;91:398-402.3. Boran P, Tokuc G, Pisgin B, Oktem S, Yegin Z, Bostan O. Efeito da obesidade na função ventilatória. J Pediatr (Rio J) 2007;83:171-6.

4. Ferraz VE. Aspectos genéticos - clínicos e laboratoriais na Síndrome de Prader Willi [tese de doutorado]. Ribeirão Preto: USP - Ribeirão Preto; 2002.

5. Goldstone AP, Thomas EL, Brynes AE, Castroman G, Edwards R, Ghatei MA et al. Elevated fasting plasma ghrelim in Prader-Willi Syndrome adults is not solely explained by their reduced visceral adiposity and insulin resistance. $J$ Clin Endocrinol Metab 2004;89:1718-26.

6. Koenig K, Klin A, Schultz R. Deficits in social attribution ability in Prader-Willi syndrome. J Autism Dev Disord 2004;34:573-82.

7. Hinton EC, Holland AJ, Gellatly MS, Soni S, Owen AM. An investigation into food preferences and the neural basis of food-related incentive motivation in Prader-Willi syndrome. J Intellect Disabil Res 2006;50:633-42.

8. Benarroch F, Hirsch HJ, Genstil L, Landau YE, Gross-Tsur V. Prader-Willi syndrome: medical prevention and behavioral challenges. Child Adolesc Psychiatr Clin N Am 2007;16:695-708. adolescentes com SPW e alguns dos achados coincidem com dados internacionais ${ }^{(13-16)}$. As alterações de comportamento detectadas nos pacientes analisados pressupõem a presença de sinais de transtornos mentais da infância e da adolescência, ou seja, transtornos de humor e de comportamento. Identificou-se, também, o agravante social destas crianças apresentarem quadros psicopatológicos sem acompanhamento de especialistas na área da saúde. Muitas dessas alterações podem prejudicar o manejo familiar e social dos pacientes. Embora a maior preocupação dos pais se concentre ao redor da hiperfagia, o atendimento exige estratégias de intervenção interdisciplinar. Os dados encontrados refletem a necessidade desse tipo de atuação.

Diferente de outros países, onde existem serviços de saúde especializados que fornecem atendimento e monitoramento para pessoas com síndromes dismórficas e deficiência mental, o grupo amostral do presente estudo não parece se beneficiar com propostas deste tipo ${ }^{(8,13-16)}$. Na tentativa de oferecer melhor assistência a tais pacientes, este estudo se constitui em uma primeira etapa do trabalho focado na avaliação para, posteriormente, possibilitar intervenção multidisciplinar junto à Clínica de Distúrbios do Desenvolvimento do Programa de Pós-Graduação em Distúrbios do Desenvolvimento do Centro de Ciências Biológicas da Universidade Presbiteriana Mackenzie. O programa prevê desenvolver, implementar e avaliar estratégias de treinamento e manejo comportamental direcionado a pais para controle de comportamentos dessas crianças e adolescentes.
9. Jauregi J, Arias C, Vegas O, Alén F, Martinez S, Copet P et al. A neuropsychological assessment of frontal cognitive functions in Prader-Willi syndrome. J Intellect Disabil Res 2007;51:350-65.

10. Dykens EM, Roof E. Behavior in Prader-Willi syndrome: relationship to genetic subtypes and age. J Child Psychol Psychiatry 2008;49:1001-8.

11. Goos LM, Ragsdale G. Genomic imprinting and human psychology: cognition, behavior and pathology. Adv Exp Med Biol 2008;626:71-88.

12. Semenza C, Pignatti R, Bertella L, Ceriani F, Mori I, Molinari et al. Genetics and mathematics: evidence from Prader-Willi syndrome. Neuropsychologia 2008;46:206-12.

13. Boer H, Holland A, Whittington J, Butler J, Webb T, Clarke D. Psychotic illness in people with Prader Willi syndrome due to chromosome 15 maternal uniparental disomy. Lancet 2002;359:135-6.

14. Nishida K, Okada Y, Mori H, Hirose A, Tanikawa T, Arao T et al. Marked weight loss in two female patients with Prader-Willi syndrome by behavioral modification and improvement of the environment. J UOEH 2006;28: 65-73.

15. Lindgren AC, Barkeling B, Hägg A, Ritzén EM, Marcus C, Rössner S. Eating behavior in Prader-Willi syndrome, normal weight, and obese control groups. J Pediatr 2000;137:50-5. 
16. Kim JW, Yoo HJ, Cho SC, Hong KE, Kim BN. Behavioral characteristics of Prader-Willi syndrome in Korea: comparison with children with mental retardation and normal controls. J Child Neurol 2005;20:134-8.

17. Gross-Tsur V, Landau YE, Benarroch F, Wertman-Elad R, Shalev RS. Cognition, attention, and behavior in Prader Willi syndrome. J Child Neurol 2001;16:288-90.

18. Rosell-Raga L. Fenotipos conductuales en el síndrome de Prader-Willi. Rev Neurol 2003;36:S153-7.

19. Must A, Dallal GE, Dietz WH. Reference data for obesity: $85^{\text {th }}$ and $95^{\text {th }}$ percentiles of body mass index (wt/ht2) and triceps skinfold thickness. Am J Clin Nutr 1991;53:839-46.

20. Achenbach TM, Rescorla LA. Manual for the ASEBA school-age forms \& profiles. Burlington, VT: University of Vermont, Department of Psychiatry; 2001.

21. American Psychiatric Association. Manual Diagnóstico e Estatístico de Transtornos Mentais. $4^{\text {a }}$ ed. Porto Alegre: Artmed; 2002.

22. McGough JJ, Loo SK, McCracken JT, Dang J, Clark S, Nelson SF et al. CBCL Pediatric Bipolar Disorder Profile and ADHD: Comorbidity and Quantitative Trait Loci Analysis. J Am Acad Child Adolesc Psychiatry. 2008;47:1151-7.
23. Bayer JK, Hiscock H, Ukoumunne OC, Price A, Wake M. Early childhood aetiology of mental health problems: a longitudinal population based study. $J$ Child Psychol Psychiatry 2008;49:1166-74.

24. Goldstone AP, Holland AJ, Hauffa BP, Hokken-Koelega AC, Tauber M; speakers contributors at the Second Expert Meeting of the Comprehensive Care of Patients with PWS. Recommendations for the diagnosis and management of Prader-Willi syndrome. J Clin Endocrinol Metab 2008;93: 4183-97.

25. Wigren M, Hansen S. ADHD symptoms and insistence on sameness in PraderWilli syndrome. J Intellect Disabil Res 2005;49:449-56.

26. Soni S, Whittington J, Holland AJ, Webb T, Maina E, Boer H et al. The course and outcome of psychiatric illness in people with Prader-Willi syndrome: implications for management and treatment. J Intellect Disabil Res 2007;51: 32-42.

27. Rinaldi AEM, Pereira AF, Macedo CS, Mota JF, Burini RC. Feeding practices and physical inactivity contributions to childhood overweight. Rev Paul Pediatr 2008;26:271-7.

28. Ruggieri VL, Arberas CL. Behavioural phenotypes. Biologically determined neuropsychological patterns. Rev Neurol 2003;37:239-53. 